TENDENCIAS

Revista de la Facultad de Ciencias

Económicas y Administrativas.

Universidad de Nariño

Vol. XVII. No. 1 - 1er. Semestre 2016,

Enero-Junio - Páginas 104-119

\title{
DIAGNÓSTICO Y CARACTERIZACIÓN DE LA MINERIA ILEGAL EN EL MUNICIPIO DE SOGAMOSO, HACIA LA CONSTRUCCIÓN DE ESTRATEGIAS PARA LA SUSTITUCIÓN DE LA MINERÍA ILEGAL
}

\author{
DIAGNOSIS AND CHARACTERIZATION OF THE ILLEGAL MINING IN \\ THE MUNICIPALITY OF SOGAMOSO, TOWARDS THE CONSTRUCTION \\ OF STRATEGIES FOR THE REPLACEMENT OF THE ILLEGAL MINING
DIAGNÓSTICO E CARACTERIZAÇÃO DE EXPLORAÇÃO MINEIRA ILEGAL NA CIDADE DE SOGAMOSO, EM DIREÇÃO A CONSTRUIR ESTRATÉGIAS PARA A SUBSTITUIÇÃO DE EXPLORAÇÃO MINEIRA ILEGAL

\author{
Por: Ana Milena Serrano - Martha Stella Martínez Bernal - Luis Alejandro Fonseca Páez
}

Doctora en Administración, Universidad Autónoma de Queretano - Docente ocasional Universidad Pedagógica y Tecnológica de Colombia, ana.serrano@uptc.edu.co

Magister en Administración, Universidad Nacional de Colombia, Docente ocasional Universidad Pedagógica y Tecnológica de Colombia, martha.martinez@uptc.edu.co

Doctorando en Ingeniería, Universidad Pedagógica y Tecnológica de Colombia, Docente Universidad Pedagógica y Tecnológica de Colombia, Alejandro.fonseca@uptc.edu.co

\section{RESUMEN}

Este artículo tiene como objetivo en primera medida describir la minería ilegal en las veredas El Pedregal y Morca ubicadas en el municipio de Sogamoso, desde la visión de los propios actores, que permita, con un equipo de trabajo, planear y desarrollar estrategias puntuales para la legalización y sustitución de la minería en esta zona del departamento de Boyacá. Mediante una metodología cuantitativa no experimental transeccional - descriptiva, y la implementación de encuesta social se logró identificar las principales situaciones donde se evidencia considerablemente el ejercicio de la minería por campesinos de la región de forma artesanal sin generar sostenibilidad desde el punto de vista técnico, financiero, legal, ambiental y de normas de seguridad, ocasionando baja productividad. Para el desarrollo de esta investigación se realizaron las siguientes fases: Diagnóstico de las condiciones actuales de la minería ilegal, se diseñaron estrategias empresariales, entre las cuales están las capacitaciones sobre la normatividad y el proceso de formalización de la minería, estrategia de asociatividad para la 
comercialización del producto y la formulación de proyectos para la sustitución de esta actividad.

Palabras clave: Minería, minería ilegal, estrategias empresariales, formalización, sostenibilidad, sustitución, minería de carbón, actividades sustitutivas.

JEL: L1, L72, Q1

\section{ABSTRACT}

This article takes as an objective in the first measurement to describe the illegal mining in el Pedregal and Morca paths located in Sogamoso city, from the vision of the proper actors, for then with a work crew, to glide and to develop some strategies for the legalization and replacement of the mining in this zone of the Boyacá department. By means of a quantitative not experimental methodology transactional descriptive, and the implementation of social poll was achieved to identify the principal situations where the exercise of the mining is demonstrated considerably by peasants of the region of handmade form without generating sustainability from the technical, financial, legal, environmental point of view and of safety norms, causing low productiveness. For the development of this investigation the following phases were realized: Diagnosis of the current conditions of the illegal mining, there were designed managerial strategies, between which the trainings are on the legal and the process of formalization of the mining, strategy of associative for the commercialization of the product and the project formulation for the replacement of this activity.

Key words: Mining, illegal mining, managerial strategies, formalization, sustainability, replacement, coal mining, substitute activities.

JEL: L1, L72, Q1

\section{RESUMO}

Este artigo tem como objetivo descrever o primeiro passo para exploração mineira ilegal nas veredas el pedregal e Morcá localizados no município de Sogamoso, a partir da perspectiva dos próprios atores, e, em seguida, com uma equipe, planejar e desenvolver algumas estratégias para legalização e substituição de mineração na região de Boyacá. Em uma metodologia quantitativa não-experimental trans-seccional - descritiva, e implementação de inquérito social foi capaz de identificar as principais situações em que o exercício da mineração evidenciados de forma dramática pelos agricultores da região com a mão, sem gerar sustentabilidade do ponto de vista técnico, financeiro, normas ambientais e de segurança jurídica, resultando em baixa produtividade. Para o desenvolvimento desta pesquisa as seguintes etapas são executadas: Diagnóstico das condições atuais de exploração mineira ilegal, estratégias de negócios, entre as quais a formação sobre a regulamentação e processo de formalização de mineração, estratégia de parceria pela comercialização do produto e formulação de projetos para substituir essa atividade.

Palavras-chave: Mineração, exploração mineira ilegal, estratégias de negócios, sustentabilidade, substituição, mineração de carvão, atividades alternativa.

JEL: L1, L72, Q1 
Ana Milena Serrano - Martha Stella Martínez Bernal - Luis Alejandro Fonseca Páez

Diagnóstico y caracterización de la mineria ilegal en el Municipio de Sogamoso, hacia la construcción de estrategias para la sustitución de la minería ilegal

\section{INTRODUCCIÓN}

La minería se define como la ciencia, las técnicas y las actividades que tienen que ver con el descubrimiento y la explotación de yacimientos minerales. De manera estricta, el término se relaciona con los trabajos subterráneos subacuáticos o a cielo abierto. En la práctica, el término incluye las operaciones a cielo abierto, canteras, dragado aluvial y operaciones combinadas que incluyen el tratamiento y la transformación. La minería es una de las actividades más antiguas, la cual consiste en la obtención selectiva de minerales y otros materiales (Ministerio de Minas y Energía, 2003, p. 108). La producción de energéticos y combustibles, ha llevado a que la explotación del carbón se incremente notoriamente en las últimas décadas, especialmente en los países en vía de desarrollo debido a que es el insumo energético más barato en el mercado.

En la actualidad, Colombia posee una abundancia de recursos naturales que debería ser la base de su distribución productiva y de su especialización para el comercio internacional. La minería en nuestro país, está considerada como una de las actividades económicas con mayor crecimiento y desarrollo en los últimos años. "Durante el año 2014, la explotación de minas y canteras registró una participación del 7,32.\% en el PIB” (Ministerio de Minas y Energía, 2014, p.4) , debido al notable impulso que ha tenido la minería del carbón desde los años ochenta, principalmente en los departamentos del Cesar y la Guajira, (UPME, 2011, p. 9), lo que ha originado ingresos significativos para el progreso del país. Según el DANE (2011), la actividad minera origina 46.627 empleos directos e indirectos. Se estima que en Colombia existen un total de 9.600 títulos mineros que ocupan entre 100.000 y 200.000 personas; el 70\% de estas son ilegales (OIT, 1999). Según la Asociación Nacional de Minería, a 2014 existían 1.828 títulos mineros para carbón y no todos ellos se encontraban en explotación. Esta tendencia económica y minera del país demuestra que existen situaciones particulares dentro del uso y comercio de la minería en los distintos departamentos, que afectan directamente el desarrollo notorio de este tipo de economía.

En departamentos como Boyacá, la economía se desarrolla desde distintas actividades realizadas por el sector de servicios, representadas en un 10\%, seguidas por la agricultura con una participación del 30\%, y la más representativa es la minería con un 50\% de contribución, siendo esta actividad la más destacada para la economía departamental. Los minerales como el hierro y la caliza son algunos de los principales productos para la industria, y las cementeras y la extracción del carbón y esmeraldas son los productos que más resaltan la economía del departamento de Boyacá (IMC, 2013, p. 2).

La minería en el departamento se ha venido posicionando como un mercado con proyección: en el departamento de Boyacá existen, basados en cifras de mayo de 2014, 1.540 títulos mineros vigentes; de los cuales 1.492 fueron fiscalizados. De los títulos fiscalizados, el 35\% está en fase de exploración, el 30\%, en construcción y montaje, y el 35\%, en fase de explotación. La producción de carbón en el departamento se concentra en 3 franjas: al sur occidente en el área Checua-Lenguazaque; en el área central en el corredor Tunja-Paipa-Duitama y al oriente en el área SogamosoJericó. Dicha actividad se concentra principalmente en las provincias de Sugamuxi, 
Alto Ricaurte y Valderrama (Pineda, 2014). Así mismo, en Boyacá existen 1.574 títulos mineros vigentes que representan un área de 318.637 hectáreas, un 14\% del departamento. El 36\% de esos títulos se encuentran en etapa de exploración, el 29\% en construcción y montaje, y el 35\% en fase de explotación” (ANM, 2014)

En Boyacá, uno de los municipios con alta actividad minera es el municipio de Sogamoso. Según estudios estadísticos del DANE, se encuentra que la actividad económica de la minería en Sogamoso se caracteriza por la explotación de minerales como carbón, arcillas, arenas, recebo y roca fosfórica, caracterizadas por ser intensivas y en general se pueden clasificar como de subsistencia y pequeña minería. El carbón que predomina en el municipio de Sogamoso es el térmico, el cual tiene bastante demanda para los procesos de las empresas industriales. En el municipio de Sogamoso existen 30 títulos mineros de carbón y 250 bocaminas ubicadas en las veredas de Morca, Ombachita, Pedregal, Primera Chorrera y las Cintas. Dentro de los principales minerales explotados en el municipio se encuentra el carbón, la arcilla, la roca fosfórica y la arena (Acuerdo Municipal No. 013-2012, 2013; Plan de desarrollo de Sogamoso, 2012).

La actividad minera en el municipio de Sogamoso al igual que en el Departamento ha tenido un desarrollo importante dentro del aparato productivo, las tendencias han presentado un aumento considerable en los últimos años, la coyuntura económica mundial, y la riqueza geológica presente, permite afirmar que el futuro cercano estará enmarcado por una ascendente actividad de explotación y comercialización de minerales.

Sin embargo, esta tendencia económica ha llevado a la proliferación de minería ilegal en el municipio, condición que es preocupante por los riesgos que asumen las personas que desarrollan esta actividad. Del mismo modo, se evidencia la falta de control en lo relacionado con la seguridad minera, ya que ellos realizan esta actividad sin los cuidados mínimos y sin las precauciones necesarias para extraer el mineral, ocasionando accidentalidad y muerte. Adicional a esto, también se puede evidenciar que la mayoría de las personas que realizan esta actividad ilícitamente no cuentan con seguridad social y seguridad ocupacional que pueda brindar el bienestar al minero, lo que ha conducido en el último año un incremento considerable de accidentalidad y muerte. Según informe de Fedesarrollo (2012) una de las causas sociales que origina la minería ilegal es por el desplazamiento de la actividad primaria como es la agricultura y ganadería, resaltando que las condiciones del campo son tan inciertas que en el momento de sacar sus productos para la venta, los precios ya presentan un valor bajo, ocasionando pérdida y endeudamiento al agricultor, en cambio la minería presenta buenos ingresos, ya que la minería se trabaja a destajo y los mineros devengan según la capacidad y la fuerza que ejerzan para extraer el producto y su salario se cancela a diario, quincenal o mensual, según acuerdo establecido por las partes.

Esta situación ha dirigido a instituciones como Fedesarollo (2011) a realizar estudios para determinar factores y perfiles psicosociales de los participantes a esta actividad productiva, y comprender así un poco más la dinámica social en relación a esta actividad explotadora de materiales y minerales. En la mayoría de los casos representan la primera generación de mineros. Hasta ahora están empe- 
Ana Milena Serrano - Martha Stella Martínez Bernal - Luis Alejandro Fonseca Páez

Diagnóstico y caracterización de la mineria ilegal en el Municipio de Sogamoso, hacia la construcción de estrategias para la sustitución de la minería ilegal

zando a vincularse los hijos de algunos de ellos; es un minero fuerte y resistente, así como dedicado a su trabajo versus los mineros de otras regiones; por ser una región donde la actividad es más reciente, se describe mayor compromiso por parte del minero con el cumplimiento de las normas y la legalidad, pues reconocen la importancia de estar en orden con las autoridades para preservar su fuente de ingreso. Rápidamente se han acogido a la normatividad y a la legalidad pese a que el proceso ha sido lento.

Dado que el volumen de mineros es pequeño y artesanal, su inicio en el negocio es muy rudimentario, no obstante, se reconoce cierto nivel de tecnificación para la explotación. El nivel de profesionalismo es muy bajo y para la mayoría no se percibe la necesidad de crecer, es solo un medio de subsistencia versus un negocio hacia el futuro; es una región que prefiere trabajar con mineros de allí mismo, que viven en sus casas cerca de la mina; solo un 20\% a 30\% vienen de otras regiones del país y viven en campamentos; personalidad/carácter: personas difíciles de organizar. Los mineros rasos no se comprometen con un horario laboral, trabajan de acuerdo con la capacidad de cada uno y con las aspiraciones económicas personales. Parte del dinero se invierte en consumo de licor y cerveza; son personas de poca fidelidad al patrón, se mueven entre las minas de la región buscando el mejor postor en pago; el propietario de la mina ha logrado superar estas condiciones tan básicas y tiene una mayor conciencia de buscar unas mejores condiciones de trabajo para el minero, pero se siente muy frustrado por la falta de compromiso del minero raso; y la mentalidad del minero es vivir al día, no se proyectan hacia el futuro, son de pocas aspiraciones, no cuentan con un proyecto de vida y de formación, solo quieren recibir su dinero de inmediato (Fedesarollo, 2011).

Estas condiciones específicas en el contexto de Boyacá, han llevado que las personas dueñas y que laboran en dichas actividad económica, carezcan en muchos casos de estrategias para la legalización y sustitución de la minería ilegal, ya sea por falta de inversión para tecnificar la extracción de este mineral o por falta de conocimiento de metodologías y normatividades para legalizar esta actividad productiva y económica del departamento. Estos aspectos han llevado a reforzar y mantener tal actividad, tanto que en municipios como Sogamoso, especialmente en las veredas el Pedregal y Morca la minería ilegal se mantiene como un ejercicio que a la luz de la legalidad será difícil normalizar.

\section{CARACTERIZACIÓN DE LA MINERÍA ILEGAL EN LAS VEREDAS EL PEDREGAL Y MORCA- SOGAMOSO}

En Sogamoso, el número de minas se encuentran ubicadas principalmente en las veredas del Pedregal y Morca. La vereda el Pedregal cuenta con un total de 12 minas y la vereda de Morca con 2 minas.

La vereda de Morca se encuentra ubicada al extremo suroriental del municipio de Sogamoso, en la actualidad cuenta con 1000 habitantes y su principal actividad económica es la extracción de carbón y la agricultura , esta vereda es reconocida a nivel nacional por ser religiosa, donde es visitada por muchos turistas de todos los lugares de Colombia. 
Tabla 1. Distribución de Minas de Carbón Veredas Pedregal y Morca Municipio de Sogamoso

\begin{tabular}{|c|l|c|}
\hline NOMBRE DE LA VEREDA & \multicolumn{1}{|c|}{ TIPO DE CARBÓN } & NÚMERO DE MINAS \\
\hline \multirow{3}{*}{ Pedregal } & Carbón térmico & 6 \\
\cline { 2 - 3 } & Carbón triturado molido & 5 \\
\cline { 2 - 3 } & Carbón coquizable metalúrgico & 1 \\
\hline Morca & Carbón térmico & 2 \\
\hline
\end{tabular}

Fuente: Catastro minero.

Figura 1. Entrada Principal Mina de Carbón Municipio de Sogamoso
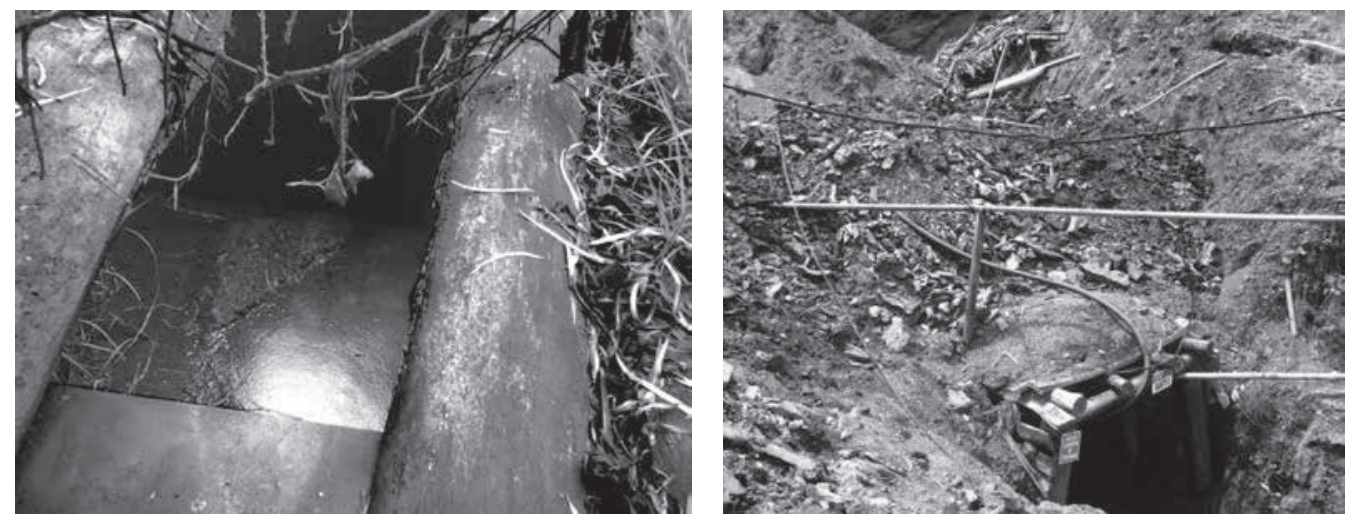

Fuente: Autores

La vereda pedregal está conformada por dos sectores Aposentos, Pedregal bajo y Pedregal Alto y la Esperanza, es una vereda caracterizada por la minería y la agricultura y en cuanto la minería predomina el carbón térmico.

Dentro de las situaciones anteriormente descritas, bajo una metodología Cuantitativa no experimental, entendida como aquella que se realiza sin manipular deliberadamente variables y no se hace modificar intencionalmente las variables independientes donde el propósito de ésta, es observar fenómenos tal y como se dan en su contexto natural, para después analizarlos, a partir de un diseño metodológico transeccional descriptivo, como aquel que tiene como objetivo indagar la incidencia y los valores que se manifiestan en una o más variables, donde el procedimiento consiste en medir en un grupo de personas u objetos una o generalmente más variables y proporcionar su descripción en un momento de tiempo determinado (Hernández, Fernández, \& Baptista, 2006), se hizo una caracterización de población a 14 minas informales, se realizó visitas de campo y encuestas a 50 personas, entre mineros propietarios de las minas ilegales ubicadas en las veredas de Morca y el Pedregal en el municipio de Sogamoso desde la información sumi- 
nistrada por la Agencia Nacional Minera. Después de tomados los datos, se hizo un análisis de las evidencias. Recopilada y analizada esta información, se diseñaron las estrategias dirigidas a los mineros de los sectores mencionados, con el fin de darles a conocer posibles soluciones a este problema, bien sea mediante la sustitución de esta actividad, o mediante el manejo y el control de la minería ilegal. Posteriormente se sensibilizó a los participantes diversas estrategias empresariales que son económicamente viables, las cuales permiten controlar o sustituir el desarrollo de la minería ilegal en el municipio de Sogamoso.

Por medio de visitas de campo a las veredas el Pedregal y Morca, y con el acompañamiento de un ingeniero de minas, los propietarios de las minas y líderes comunales, se implementó un instrumento tipo encuesta social que permitió hallar las siguientes situaciones:

Figura 2. Tiempo de realización de la minería ilegal

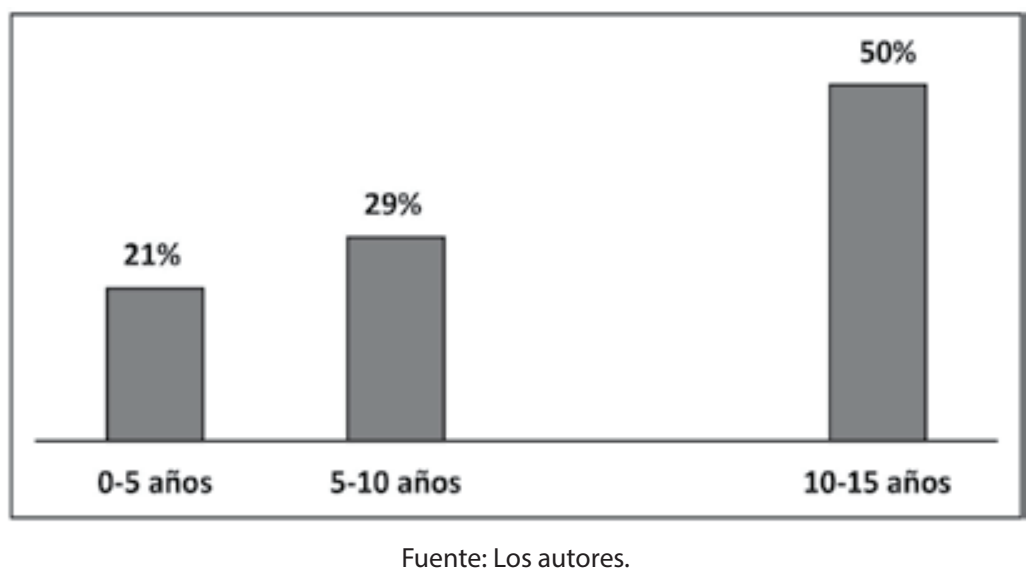

Frente al tiempo de realización de minería ilegal, en la Figura 2 se puede evidenciar que el $21 \%$ de las minas realizan esta actividad sin ningún control, aproximadamente desde hace 0-5 años. Esto surge por la falta de vigilancia de las entidades gubernamentales. El 29\% viene efectuando esta actividad en un periodo de 5-10 años, lo cual se presenta por falta de gestión por parte de los dueños de las minas, que no están interesados en legalizar sus títulos mineros a causa de los costos que esto genera. El 50\% de las personas encuestadas se dedican a esta labor desde hace 10-15 años, situación que es provocada porque las solicitudes de legalización que se han hecho, se han negado por diversas causas, entre las cuales se encuentra la falta de la licencia ambiental y de cumplimiento de la normatividad minera. Lo anterior ha ocasionado deterioro ambiental, social y económico al municipio de Sogamoso.

En cuanto al mineral y material que más se explota sin ningún control, se encuentra lo siguiente: el 93\% de los encuestados explota carbón, el cual es de 
fácil adquisición y de mayor consumo por las empresas de la región, ya que la gran mayoría de las industrias sogamoseñas se benefician de este mineral y un 7\% corresponde a la explotación de arena, teniendo en cuenta que la ciudad de Sogamoso presenta el sector de la construcción con un importante crecimiento.

Figura 3. El mineral que más se explota

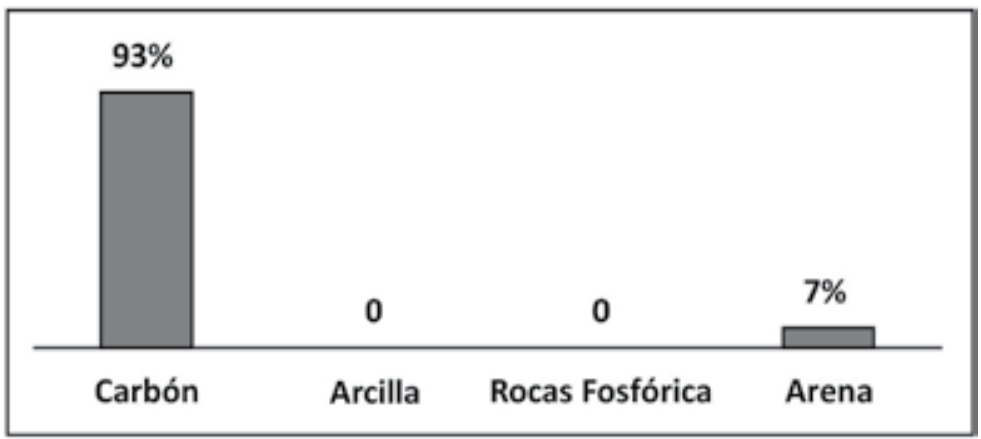

Fuente: Los autores

Figura 4. Causas de la explotación de la minería ilegal

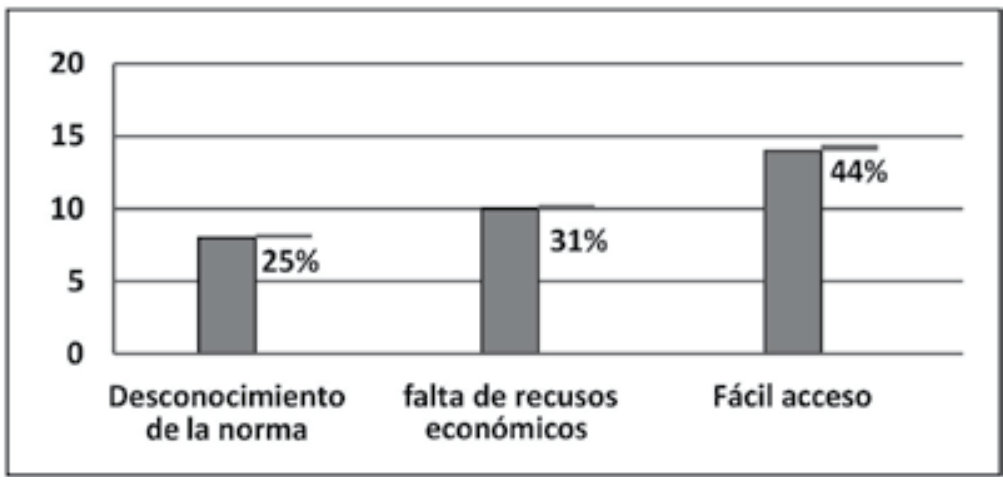

Fuente: Los autores

El 25\% de los mineros encuestados que se dedica a esta actividad no conoce la normatividad que establece el Estado colombiano, ya que esta población tiene un bajo nivel de escolaridad. Según la encuesta, el 31\% menciona que no cuenta con recursos financieros para hacer la tramitología, el 44\% practica la minería ilegal por el fácil acceso y ubicación de las minas a sus viviendas y porque no tienen que incurrir en gastos administrativos para su realización.

Referente a la necesidad de sustituir la minería ilegal por otras actividades económicas, el 43\% de los encuestados manifestó su deseo de iniciar todo el proceso 
de legalización o de sustituirla por la agricultura, ya que Sogamoso cuenta con la diversidad geográfica para iniciar otro tipo de labor. El 23\% se inclina por la cría y comercialización de la trucha arcoíris, esta opción se origina por la cercanía que tiene el municipio con la laguna de Tota (Aquitania). El 21\% se inclina por la posibilidad de solicitar ayuda a las entidades competentes del Estado.

Figura 5. Tipo de estrategia para sustituir y/o legalizar la minería ilegal

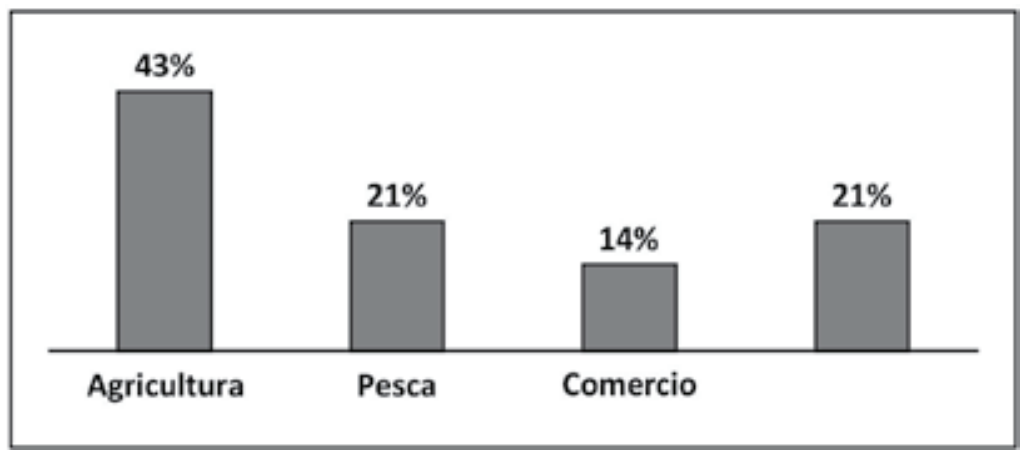

Fuente: Los autores

Con la aplicación de esta encuesta social se pueden identificar las debilidades, fortalezas, amenazas y oportunidades que tienen los mineros, considerando el análisis PEST donde se encuentran los factores económicos, jurídicos, sociales y tecnológicos para el desarrollo de esta actividad.

El aspecto económico que se evidenció por la práctica de esta minería ilegal es preocupante, debido que estas personas al actuar al margen de la ley están evadiendo el pago de los impuestos que origina el ejercicio legal de la minería. Los propietarios de las minas no invierten en ningún tipo de asesoría ni planeación para el desarrollo de la minería. Adicionalmente se puede notar que la mayoría de las personas que se dedican a esta actividad ilícita, no cuenta con seguridad social y seguridad ocupacional que pueda brindar el bienestar a la vida del minero, lo que ha llevado en el último año a un incremento considerable de accidentalidad y muerte de trabajadores mineros en el Departamento de Boyacá.

En cuanto a los factores tecnológicos, se puede comprobar que estas minas no cuentan con tecnología adecuada para esta práctica, lo cual genera atraso y un aumento de accidentalidad y bajo nivel de productividad, debido a que la minería ilegal se realiza artesanalmente, se utilizan herramientas de baja calidad, las cuales no cumplen con las exigencias para practicar este oficio.

La normatividad jurídica colombiana relativa a la legalización de la minería, es muy exigente, lo cual ha hecho que en la práctica, los trámites de formalización demoren muchos años ocasionando desánimo en los mineros. El Estado hace unos requerimientos muy costosos para estos mineros que, en su mayoría están dedicados a esta actividad para sobrevivir, de tal manera que ellos no tienen los recursos económicos para realizar todos los trámites. Además, como estas prácticas mineras 
son tan rudimentarias y ocasionan tantos daños ambientales, cuando el minero logra presentar la documentación para solicitar la licencia ambiental, esta le es negada, impidiéndole así la formalización, puesto que sin la licencia no hay autorización para la iniciación de los trabajos y obras de explotación minera.

Los factores sociales y demográficos son esenciales para el municipio, puesto que la minería ilegal genera consecuencias sociales como la pobreza, la violencia, la deserción escolar y en algunos casos, la muerte.

En el aspecto ambiental, se observó que en las zonas donde se practica la minería ilegal se está presentando contaminación del suelo, debido a la generación de residuos sólidos como grasas y aceites de maquinaria y vehículos; contaminación del agua, ya que en estas veredas se encuentran fuentes hídricas; enorme deforestación de la vegetación, por causa de las excavaciones que allí se adelantan; además se está ocasionando la deforestación, lo cual está impidiendo que el sector de la agricultura inicie procesos de cultivos.

En el aspecto sociocultural, se comprobó que muchos trabajadores ejercen esta actividad por tradición familiar y porque ellos desde niños desempeñan esta labor, en otros casos se detectó que los mineros sustituyeron la agricultura por la minería ilegal debido a la facilidad y las condiciones económicas, ya que ellos no reciben el salario diario en la actividad agrícola.

La infraestructura física de las minas es muy rudimentaria, las personas que laboran en estas minas utilizan herramientas de trabajo manuales sencillas, como La Barra, cacho o Batea lo que la hace inadecuada para desarrollar, por ejemplo, proyectos sostenibles o mayores negocios (esto en el evento de conseguir la legalización), sumado a la falta de conocimientos y habilidades para ofrecer un producto bajo ciertos parámetros de calidad, de manera permanente, oportuna y a un costo determinado. Hay que recordar que este grupo de mineros presenta un bajo nivel de escolaridad

Como una generalidad de las personas que realizan actividades económicas informales, en este tipo de trabajo existe también acceso restringido a productos y servicios financieros: sin crédito, los productores de bajos recursos no pueden financiar sus eventuales proyectos, y sin seguros, no pueden proteger sus pocas pertenencias e ingresos contra crisis como enfermedades, desastres naturales o robos. Algunos mineros comentaron que al tratar de solicitar un crédito bancario, el banco les hizo unas exigencias que ellos jamás podrían cumplir, condición que la implementación de proyectos, especialmente los que incluyen un componente de negocio, supone la existencia de confianza entre los actores que participan en él, lo que asegura su viabilidad y permanencia en el tiempo. Sin embargo, en el medio de los mineros ilegales de Sogamoso, la descomposición social causa inseguridad física, económica e institucional y genera desconfianza entre actores, constituyéndose en una barrera antropológica que desanima y obstaculiza la realización de negocios. Como ya se mencionó, los mineros desconfían del Estado, y además desconfían de los otros mineros para establecer asociaciones y desarrollar negocios conjuntamente. 
Ana Milena Serrano - Martha Stella Martínez Bernal - Luis Alejandro Fonseca Páez

Diagnóstico y caracterización de la mineria ilegal en el Municipio de Sogamoso, hacia la construcción de estrategias para la sustitución de la minería ilegal

\section{PLANEACIÓN Y DESARROLLO DE ESTRATEGIAS PARA LA SUSTITUCIÓN DE LA MINERÍA ILEGAL}

A partir de este panorama y su análisis, se decidió dentro del proceso de inmersión a la problemática de las veredas el Pedregal y Morca, con el equipo de profesionales conformado por psicólogos, ingenieros de minas, administradores de empresas , presidentes de las juntas de acción comunal y dueños y trabajadores de las minas, constituir variadas estrategias para la sustitución de la minería ilegal, con las que se busca que los mineros den un inicio para la legalización de la explotación minera del carbón de esta región. Para sustituir esta actividad por otras propuestas económicas el investigador y equipos de trabajo optó por desarrollar las siguientes estrategias con la población foco de este análisis en este documento:

a) La capacitación.

b) Estrategia de asociatividad.

c) Sustitución de la minería ilegal por otras actividades económicas.

Las estrategias de capacitación son definidas por Perozo (2004), son formas de gestión de conocimiento que ayuda a las organizaciones a la innovación y estimula la adquisición de conocimiento, donde se involucran la ética y los valores de la comunidad.

En el entorno de las organizaciones, el contexto de capacitación deja de ser una actividad secundaria para convertirse en una facultad crítica en las estrategias de competitividad (Guinazú, 2004) citado por Matlay (2010, p. 337-342), asegura que la iniciativa de la capacitación es importante para las pequeñas empresas y debe ser dirigida a las necesidades específicas de los propietarios de estas. Para los propósitos de este trabajo, la capacitación estaría orientada a informar a los mineros sobre los trámites necesarios para la legalización de su actividad y las responsabilidades que ellos deben asumir, toda vez que la formalización no es solo tener el título minero, pues implica también responsabilidades económicas, ambientales, de seguridad y de compromiso con la sociedad. $\mathrm{O}$, si el minero está más interesado en dedicarse a una actividad diferente, entonces la capacitación debe orientarse a la preparación de éste, para asumir su nueva forma de vida, con todo lo que esto implica: dónde adquirir conocimientos de un nuevo oficio, instituciones a las cuales acudir para solicitar apalancamientos financieros y asesoría técnica durante todo este proceso.

Frente a esta estrategia, se dieron capacitaciones sobre motivación, teniendo en cuenta que la mayoría de los mineros hacen esta labor por circunstancias forzosas y no porque realmente quieran ejercer este oficio, debido a las condiciones precarias con las que llevan a cabo esta actividad. El evento se llevó a cabo en el municipio de Sogamoso y tuvo el acompañamiento de un ingeniero de minas, un psicólogo, un abogado y un administrador de empresas, los cuales guiaron el proceso de las sesiones taller.

Se inició la sesión dando a conocer la importancia de la solución de la minería ilegal, orientando sobre los beneficios económicos que obtendrían los mineros que ejercen esta actividad y el municipio por el pago de las regalías. Se hizo énfasis en las exigencias de las autoridades mineras sobre el cumplimiento de la normatividad 
y así evitar la suspensión de esta actividad. El psicólogo promovió la motivación, mostrando qué tipo de población y las características sociales, familiares y económicas que presentan los mineros que están realizando esta labor. Él fue el encargado de hacer una reflexión acerca de la importancia de la prevención y ejecución de esta actividad con todos los cuidados exigidos por el Estado colombiano. El ingeniero de minas estuvo encargado de explicar toda la normatividad ambiental y minera haciendo énfasis en todo lo relacionado con el contrato de concesión, los trámites que deben seguir para hacer la solicitud del contrato y las diferentes herramientas que utiliza Ingeominas para hacer el proceso de legalización. En lo referente al aspecto ambiental, explicó toda la tramitología que se debe seguir para la obtención de las licencias ambientales, así mismo expuso que la minería se debe llevar a cabo con un planeamiento y debe ser sostenible desde el punto de vista técnico, económico, jurídico, ambiental y empresarial.

El administrador de empresas fue el encargado de dar a conocer las diferentes alternativas económicas que pueden desarrollar para la sustitución de esta actividad, les informó de todos los medios de financiación que se tienen en Colombia para el apoyo de estos proyectos, y adicionalmente hizo una capacitación acerca de emprendimiento, apoyándose en la ley 1014 que así lo promueve y posteriormente les habló de la motivación, haciendo énfasis en la importancia de legalizar la minería o de sustituir esta actividad por otras que les puedan generar beneficios económicos, sociales y ambientales.

Otra estrategia utilizada y socializada con los mineros de estas veredas fue la asociatividad, que según Cervilla (2007, p. 230-248), es un "mecanismo de cooperación entre empresas pequeñas y medianas donde cada empresa decide participar voluntariamente con los otros participantes para la búsqueda de un objetivo común”. Frente a la situación económica que están viviendo los mineros en esta región de Boyacá, es importante llegar a esta asociatividad empresarial, teniendo en cuenta que esta es entendida como "la cooperación directa e implícita a través de canales informales, las relaciones bilaterales y multinacionales directas con otras empresas como proveedores, compradores y el estado" (Kingsbury \& Hayter, 2006, p. 596609). La asociatividad es vista como un modelo que permite "la implementación de estrategias colectivas y de carácter voluntario con el fin de alcanzar niveles de competitividad" (Liendo \& Martínez, 2001, p. 311-319). Uno de los objetivos de la asociatividad es aprovechar las fortalezas de cada miembro y así minimizar los riesgos individuales.

La estrategia de asociatividad se implementará una vez sean aceptadas las condiciones jurídicas para poder operar desde la formalización minera, se les propondrá a los dueños de las minas que se asocien con el fin de conformar una empresa minera sostenible en el municipio de Sogamoso, y así comercializar los minerales y materiales de carbón bajo los estándares establecidos en Colombia. En este punto es importante tener en cuenta lo expresado aquí en cuanto a las restricciones, ya que antes de iniciar proyectos asociativos es importante trabajar la parte relativa a la confianza entre los actores.

Para la sustitución de la minería ilegal por otras actividades económicas, se tomó como referencia el acuerdo municipal número 013-2012, que contiene el Plan de 
Ana Milena Serrano - Martha Stella Martínez Bernal - Luis Alejandro Fonseca Páez Diagnóstico y caracterización de la mineria ilegal en el Municipio de Sogamoso, hacia la construcción de estrategias para la sustitución de la minería ilegal

Desarrollo, periodo 2012-2015 de Sogamoso (2013, p. 164-193), suministrado por la alcaldía, donde se observa que el sector primario de la economía del municipio se centra en la agricultura, pesca y minería. Con esta información se diseñaron estrategias empresariales y se plantearon proyectos con el sector que tiene más demanda en la economía del municipio, en este caso la agricultura. Otra estrategia empresarial que se diseñó es la cría y comercialización de la trucha arcoíris.

La formulación de proyectos se basó desde los componentes que establecen la estrategia empresarial: a) Los objetivos de la acción empresarial claramente definidos (atributos, escalas de medida, normas, horizonte temporal). b) El plan de acción en el ámbito de la empresa total y en el ámbito de las divisiones. c) Los programas funcionales que describen y miden las consecuencias del plan para cada una de las funciones de la empresa (finanzas, mercadeo, personal, etc.). d) Los recursos requeridos para llevar a cabo los programas (www.virtual.unal.edu.co).

Croteau y Bergeron (2001, p. 77-99) sostienen que la estrategia empresarial es vista como el "resultado de decisiones tomadas para guiar a la organización". La estrategia se puede definir como "planes que tienen los directivos superiores para obtener resultados compatibles con las misiones y objetivos de la organización y como un plan, al equivocar una orientación, una guía o un rumbo de acción hacia el futuro, es parte de un proceso de carácter cíclico, puede ser de carácter informal, intuitivo siguiendo un proceso repetitivo simple en el cual se planean objetivos" (Mintzberg \& Lampel, 2003, p. 4-150).

Según Montoya (2009, p. 23-44), la estrategia consiste en "la elección de una posición valiosa y única afianzada o enraizada en sistemas de actividades que son muy difíciles de evaluar". Este autor analiza la estrategia teniendo como referencia a Minztzberg, el cual resalta que la estrategia es importante para el desempeño y llegar al cumplimiento de las "metas", logrando con esto una "ventaja competitiva" en el estudio que se esté realizando.

De acuerdo con Manso (2003, p. 10), la formulación de las estrategias debe llevarse a cabo después de "haber identificado, evaluado y seleccionado diferentes cursos de acción", los cuales se desarrollan en cuatro pasos: a) Establecimiento de objetivos; b) Identificación de brechas; c) Formulación de cursos de acción; y d) Determinar el grado de eficiencia.

Amaya (2007, p. 8) señala que la formulación estratégica debe "convertirse en planes de acción concretos, con definición de responsables, para ello se debe proyectar el tiempo para cada uno de los proyectos estratégicos, definir los objetivos de las estrategias de cada área funcional dentro de los proyectos, así como diseñar planes de acción concretos".

Para la sustitución de la minería ilegal se formularon algunos proyectos, los cuales deben contener:

- Identificación del producto al cual se le va a hacer el estudio, y un reconocimiento del entorno.

- Elaboración de un estudio de mercado que promueva estrategias de comercialización y fortalecimiento de la estrategia empresarial en el municipio. 
- Realización de un estudio técnico, que permita tener claridad del producto y de la cantidad que se va a producir para iniciar su comercialización.

- Establecimiento de un monto de inversión para cada alternativa.

Las alternativas que se plantearon fueron las siguientes:

- Formulación y evaluación del proyecto: cultivo de papa.

- Formulación y evaluación del proyecto: cría de trucha arcoíris.

- Formulación y evaluación del proyecto: cultivo de cebolla.

\section{CONCLUSIONES}

- La minería tiene vital importancia para Colombia, y si se administra debidamente, ésta ofrece la oportunidad de promover un desarrollo de base amplia y reducir la pobreza. Sin embargo, al país todavía le queda un largo camino por recorrer para lograr minimizar sus efectos medioambientales y sociales negativos y maximizar los efectos económicos positivos de la minería.

- La falta de conocimiento de los trabajadores mineros en aspectos relacionados con su actividad, hacen de esta un trabajo de alto riesgo para la salud de los que en ella intervienen, de poca aceptación entre la comunidad que se encuentra en su entorno y de baja aplicación de conocimientos empresariales, que afectan de una u otra manera la competitividad del sector.

- Con esta investigación, se detectaron algunas de las causas de la minería ilegal, como la ausencia de apoyo por parte de las entidades del Estado y la falta de recursos económicos para iniciar el proceso de legalización.

- Después de presentar los talleres de motivación, se pudo evidenciar que los dueños de las minas tomaron conciencia respecto de la importancia de las estrategias empresariales enseñadas, por tal razón es fundamental contar con el apoyo de las entidades competentes para lograr la implementación de las mismas.

\section{RECOMENDACIONES}

Atendiendo el diagnóstico y el análisis que se efectuó con esta investigación, es importante dar a conocer a la alcaldía municipal los resultados obtenidos, con el propósito de solicitar apoyo económico y social para lograr la puesta en marcha de estas estrategias y así ayudar a esta comunidad, que se encuentra vulnerable y desamparada.

Es pertinente pedir a la alcaldía municipal el diseño de un sistema de información que permita detectar el desarrollo de la minería ilegal, con el propósito de hacer un seguimiento estricto con el apoyo de las autoridades competentes, para brindar al minero el apoyo logístico de legalización minera y licenciamiento ambiental. 
Es de suma importancia que las entidades gubernamentales encargadas del control de la minería realicen campañas de capacitación a los mineros del municipio, con el fin de mostrarles las ventajas competitivas, económicas y ambientales que tendrían si realizan esta labor de la minería de acuerdo con la normatividad vigente.

Es importante establecer alianzas entre las entidades gubernamentales y los propietarios de las minas con el fin de desarrollar diversas actividades que los beneficien.

Teniendo en cuenta que la minería es una de las actividades más importante en el municipio de Sogamoso, es de suma urgencia detectar toda la minería ilegal que está desarrollando, y así evitar el incremento de los problemas sociales, ambientales y económicos que se manifiestan debido al ejercicio indiscriminado y sin control de esta actividad.

Se requiere identificar los actuales procesos técnicos, realizar capacitaciones teóricas y prácticas a través de procesos demostrativos donde se muestran las nuevas tecnologías y metodologías para mejorar la extracción del carbón y reducir así la contaminación ambiental.

Es importante dar a conocer a la comunidad del municipio de Sogamoso que la minería ilegal, no es la única fuente de ingresos que existe, que vale la pena incentivar a las personas desde los establecimientos educativos a formarse en escenarios estratégicos donde se centra la motivación, emprendimiento empresarial y capacitación, con el fin de formar líderes que ayuden a crear empresas y así lograr la solución de la sustitución de la minería ilegal, con el apoyo de diversas entidades del Estado encargadas de emprendimiento empresarial. 


\section{REFERENCIAS}

1. AMAYA, Jairo (2007). Gerencia, planeación y estrategia. Bucaramanga: Universidad Santo Tomás.

2. ASOCIACIÓN NACIONAL MINERA. (20 de Junio de 2014). Agencia Nacional Minera. Recuperado el 15 de Mayo de 2015, de Agencia Nacional Minera : http://www.anm.gov.co/?q=Boyaca_avanza_en_Formalizacion_Minera

3. CERVILLA DE OLIVIERI, M. A. (2007). Estrategias para el desarrollo empresarial: asociatividad en el sector plástico venezolano. Revista de Ciencias Sociales (Ve), XIII (2), 230-248. Recuperado de http://www.redalyc. org/articulo.oa?id=28011677004

4. CÓDIGO DE MINAS (2001). Ley 685 Artículo 159. Bogotá

5. CROTEAU \& BERGERON. (2001). Una trilogía de tecnología de la información: la estrategia empresarial, el despliegue tecnológico y desempeño de la organización. El Diario de Sistemas de Información Estratégica, $10(2), 77-99$.

6. DANE (2011). Departamento Administrativo Nacional de Estadística - Colombia

7. FEDESARROLLO (2011, dic.). Pequeña y mediana minería de carbón del interior del país: alternativa de comercialización y financiación a partir de la conformación de alianzas estratégicas. Recuperado de http:// www.fedesarrollo.org.co/wp-content/uploads/2011/08/Peque\%C3\%B1a-y-mediana-miner\%C3\%ADade-carb\%C3\%B3n-del-interior-del-pa\%C3\%ADs-Informe-final-19-de-diciembre.pdf

8. LIENDO \& MARTÍNEZ (2001). Asociatividad. Una alternativa para el desarrollo y crecimiento de las pymes. Revista Sextas Jornadas "Investigaciones en la Facultad" de Ciencias Económicas y Estadística, 311-379. Recuperado de http://www.fcecon.unr.edu.ar/web/sites/default/files/u16/Decimocuartas/Liendo,\%20 Martinez_asociatividad.pdf

9. MINISTERIO DE MINAS Y ENERGIAS (2014). Ministerio de Minas y Energias. Recuperado el 1 de junio de 2015, de Ministerio de Minas y Energias: http://www.minminas.gov.co/documents/10180/558364/PibIVTrimestre2014.pdf/2e08741f-fa27-4e0f-81f4-fcb136886eb4

10. HERNÁNDEZ, FERNÁNDEZ \& BATISTA (2006, abril). Metodología de la investigación (4 ed.). México: McGraw Hill.

11. KINGSBURY \& HAYTER (2006, JULIO). Asociaciones empresariales y el desarrollo local: la respuesta de la industria del vino de Okanagan al TLCAN. Geoforum, 37 (4), 596-609. Recuperado de Http://www.sciencedirect.com/science/article/pii/S0016718505001375

12. MANSO (2003). Diccionario enciclopédico de estrategia empresarial. Madrid: Ediciones Díaz de Santos.

13. MATLAY, H. (2010). Formación en las pequeñas empresas En: P. Peterson, E. Baker \& B.

14. MINISTERIO DE MINAS Y ENERGÍA (2003, agosto). Glosario Minero. Bogotá: s.n.

15. MINTZBERG \& LAMPEL (2003). Safari de la estrategia. Buenos Aires: Granica.

16. MONTOYA (2009). La formación estratégica en Mintzberg y la posibilidad de una aportación para el futuro. Facultad de Ciencias Administrativas y Contables Universidad Nacional de Colombia, XVII (2), 23-44

17. OIT. Programa de actividades sectoriales. Los problemas sociales y laborales en las explotaciones mineras pequeñas. Ginebra; mayo de 1999.

18. PLAN DE DESARROLLO DE SOGAMOSO - Periodo 2012-2015.

19. PEROZO, M. (2004). Gestión del conocimiento en la capacitación para la innovación. Revista Venezolana de Análisis de Coyuntura, X (2), 117-129.

20. RUIZ, F. P. (12 de diciembre de 2014). Corporación Viva la Ciudadanía Opiniones Recuperado el 12 de mayo de 2015, de Corporación Viva la Ciudadanía Opiniones http://viva.org.co/cajavirtual/svc0429/pdfs/Articulo746_429.pdf 